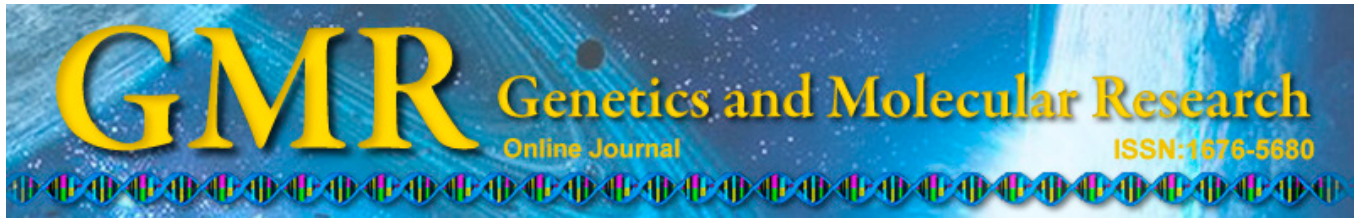

\title{
Analysis of key genes and modules during the courses of traumatic brain injury with microarray technology
}

\author{
X.-Y. Zhang1*, C.-G. Gu' ${ }^{2 *}$ J.-W. Gu ${ }^{3}$, J.-H. Zhang ${ }^{1}$, H. Zhu', \\ Y.-C. Zhang ${ }^{1}$, J.-M. Cheng ${ }^{1}$, Y.-M. Li ${ }^{1}$ and T. Yang ${ }^{1}$ \\ ${ }^{1}$ Department of Neurosurgery, ChengDu Military General Hospital, Chengdu, \\ Sichuan, China \\ ${ }^{2}$ Clinical Laboratory Department, 452 Hospital of PLA, Chengdu, \\ Sichuan, China \\ ${ }^{3}$ Department of Neurosurgery Air Force General Hospital of PLA, \\ Beijing, China \\ *These authors contributed equally to this study. \\ Corresponding author: J.-W. Gu \\ E-mail: jian-wengu@hotmail.com
}

Genet. Mol. Res. 13 (4): 9220-9228 (2014)

Received October 17, 2013

Accepted September 29, 2014

Published November 7, 2014

DOI http://dx.doi.org/10.4238/2014.November.7.9

\begin{abstract}
Gene expression data acquired at different times after traumatic brain injury (TBI) were analyzed to identify differentially expressed genes (DEGs). Interaction network analysis and functional enrichment analysis were performed to extract valuable information, which may benefit diagnosis and treatment of TBI. Microarray data were downloaded from Gene Expression Omnibus and pre-treated with MATLAB. DEGs were screened out with the SAM method. Interaction networks of the DEGs were established, followed by module analysis and functional enrichment analysis to obtain insight into the molecular mechanisms. A total of 39 samples at six time points ( $30 \mathrm{~min}, 4,8,24,72$ h, and 21 days) were analyzed and generated 377 DEGs. Eight modules
\end{abstract}


were identified from the networks and network ontology analysis revealed that cell surface receptor-linked signaling pathway, response to wounding and signaling pathway were significantly overrepresented. Altered risk genes and modules in TBI were uncovered through comparing the gene expression data acquired at various time points. These genes or modules could be potential biomarkers for diagnosis and treatment of TBI.

Key words: Traumatic brain injury; Time series; Risk genes; Network modules

\section{INTRODUCTION}

Traumatic brain injury (TBI), also known as intracranial injury, is a type of brain injury caused by external pressure, such as shock, explosion (Rehman et al., 2008) infection, or stroke. It can be divided into closed injury and open injury (Maas et al., 2008). Due to limitations of current technologies, it is not possible to detect temporary or permanent loss of function as well as structural damage (Parikh et al., 2007). Therefore, discovery of biomarkers indicating the progression of TBI is rather necessary.

A number of studies have been carried out to describe or disclose the changes. Long et al. (2003) found 253 differentially expressed genes (DEGs) in traumatically injured mouse hippocampus. Michael et al. (2005) analyzed the gene expression profile of TBI in humans and identified 104 DEGs. Besides, microRNA expression profile is also taken into consideration (Lei et al., 2009). Clear progression has also been made in the treatment of TBI. To date, neuroprotection has been regarded as an important part of the treatment of TBI (Faden, 2002). Di Giovanni et al. (2005) reported that cell cycle inhibition is an effective way to decrease both neuronal cell death and reactive gliosis after experimental TBI.

However, the process of TBI is dynamic, which involves a range of genes working at different times. Therefore, microarray time-course experiment is rather favorable and necessary in this field. It can not only detect thousands of genes, but also reflects the dynamic changes in gene expression. There are several methods to analyze the data, such as STEM (short time-series expression miner) (Ernst et al., 2005; Ernst and Bar-Joseph, 2006) GeneTS (Schafer and Strimmer, 2005; Opgen-Rhein and Strimmer, 2007), TANOVA (time-course analysis of variance) (Zhou et al., 2010) and GQL (graphical query language) (Costa et al., 2005), and these methods have their advantages and disadvantages. Here, we propose a modular analysis method, combining protein-protein interaction information and DEGs, to identify significant disturbed modules associated with TBI, followed by functional enrichment analysis to reveal their biological functions and uncover the underlying mechanisms in the progression of TBI. Our findings can help in developing new ways of diagnosis and treatment of TBI.

\section{MATERIAL AND METHODS}

\section{Microarray data}

The microarray data set GSE2392 (Natale et al., 2003) was obtained from Gene Ex- 
pression Omnibus (GEO, http://www.ncbi.nlm.nih.gov/geo/). Raw data were collected with information of the platform GPL85 (Affymetrix Rat Genome U34 Array). Totally, 39 mouse samples were included at six time points: $30 \mathrm{~min}, 4,8,24,72 \mathrm{~h}$, and 21 days after moderate levels of lateral fluid percussion-induced brain injury in rats. MATLAB was chosen for data pre-treatment and the average value was given for probes corresponding to one gene. Missing values were filled in with the K-means method.

\section{Screening of DEGs}

Differential analysis was conducted using the SAM (significance analysis of microarrays) method (Tusher et al., 2001) in MEV (MultiExperiment Viewer) (Saeed et al., 2003). Delta of 2 was set as the cut-off criterion to determine DEGs.

\section{Establishment of interaction networks}

Collaborations between genes and pathways are the basis of biological functions. Similarly, pathogenesis of disease can be attributed to interactions between a number of genes. Therefore, interaction networks of DEGs were established, from which global alterations were observed and investigated. First, information about protein-protein interaction was downloaded from I2D (http://ophid.utoronto.ca/ophidv2.204/) (Brown and Jurisica, 2005, 2007). Next, self-interactions and duplicates were removed and UniProtKB was converted to Entrez Gene ID with ID mapping in Uniprot (Apweiler et al., 2004). To extensively mine disease-related modules, loose criteria were applied in the present study to filter interactions.

\section{Module mining and functional annotations}

The network was visualized with Cytoscape (Shannon et al., 2003; Smoot et al., 2011). The topological property was analyzed with its plug-in Network Analysis. The modules were then mined with MCODE and functional annotations were given by network ontology analysis (NOA, http://app.aporc.org/NOA/index.html) (Wang et al., 2011).

\section{RESULTS}

\section{Identification of DEGs}

Differential analysis was conducted with the SAM method for gene expression profiles obtained at 6 time points in TBI, and Delta $=2$ was chosen as the threshold (Figure 1). A total of 377 DEGs were identified, 235 upregulated and 142 downregulated (Table 1). Only a few DEGs were obtained at the first and last time points, while the number of DEGs was the highest at $8 \mathrm{~h}$ (Figure 2). This was in accordance with clinical observation, suggesting that our results were highly reliable. 


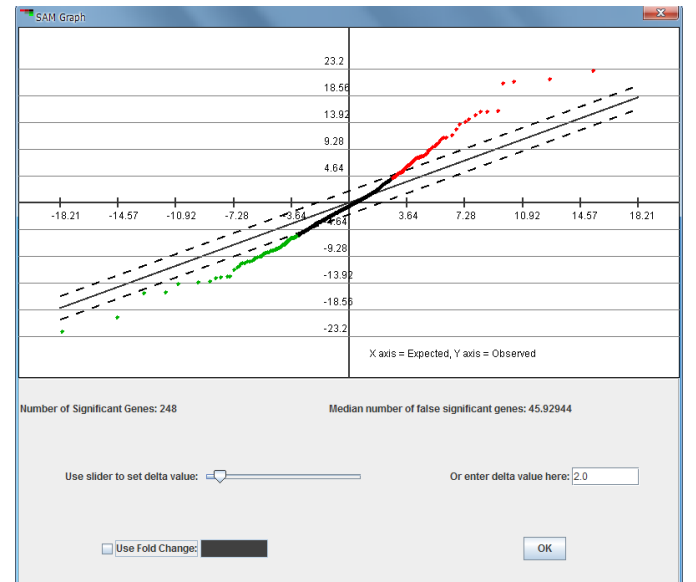

Figure 1. Differentially expressed genes (DEGs) screened out by SAM method. Downregulated DEGs were in green, upregulated in red and non-DEGs in black.

Table 1. Number of DEGs at different time points of TBI.

\begin{tabular}{lccr}
\hline Time point & Upregulated & Downregulated & Total \\
\hline $0.5 \mathrm{~h}$ & 10 & 0 & 10 \\
$4 \mathrm{~h}$ & 25 & 3 & 28 \\
$8 \mathrm{~h}$ & 145 & 103 & 248 \\
$24 \mathrm{~h}$ & 35 & 23 & 58 \\
$72 \mathrm{~h}$ & 20 & 13 & 33 \\
21 days & 10 & 0 & 10 \\
Total & 235 & 142 & 377 \\
\hline
\end{tabular}

DEGs = differentially expressed genes; TBI = traumatic brain injury.

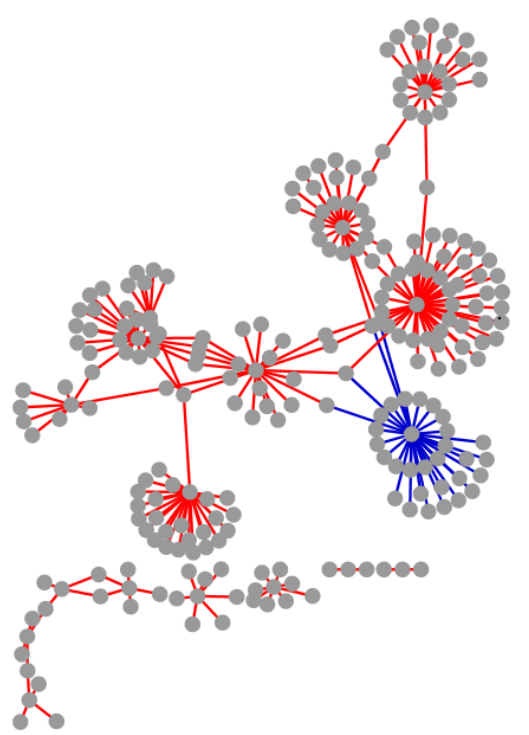

Figure 2. Interaction network for DEGs at 4-h circles represent genes and edges represent interactions = upregulated in red and downregulated in blue. 


\section{Interaction networks for DEGs}

On the basis of the information collected from I2D, 6 interaction networks were established for DEGs at various time points. The topological properties were calculated and are shown in Table 2.

Table 2. Topological properties of the 6 interaction networks.

\begin{tabular}{lcccc}
\hline Time point & No. of nodes & No. of edges & No. of upregulated edges & No. of downregulated edges \\
\hline $0.5 \mathrm{~h}$ & 317 & 358 & 358 & 0 \\
$4 \mathrm{~h}$ & 244 & 252 & 220 & 32 \\
$8 \mathrm{~h}$ & 2066 & 4542 & 3059 & 1483 \\
$24 \mathrm{~h}$ & 770 & 1031 & 903 & 128 \\
$72 \mathrm{~h}$ & 512 & 616 & 402 & 214 \\
21 days & 270 & 281 & 281 & 0 \\
\hline
\end{tabular}

\section{Results of module mining}

Modules in the network were mined with MCODE of Cytoscape and several significant modules were acquired at 8 and $72 \mathrm{~h}$. Two typical modules obtained from time point $8 \mathrm{~h}$ are shown in Figure 3.
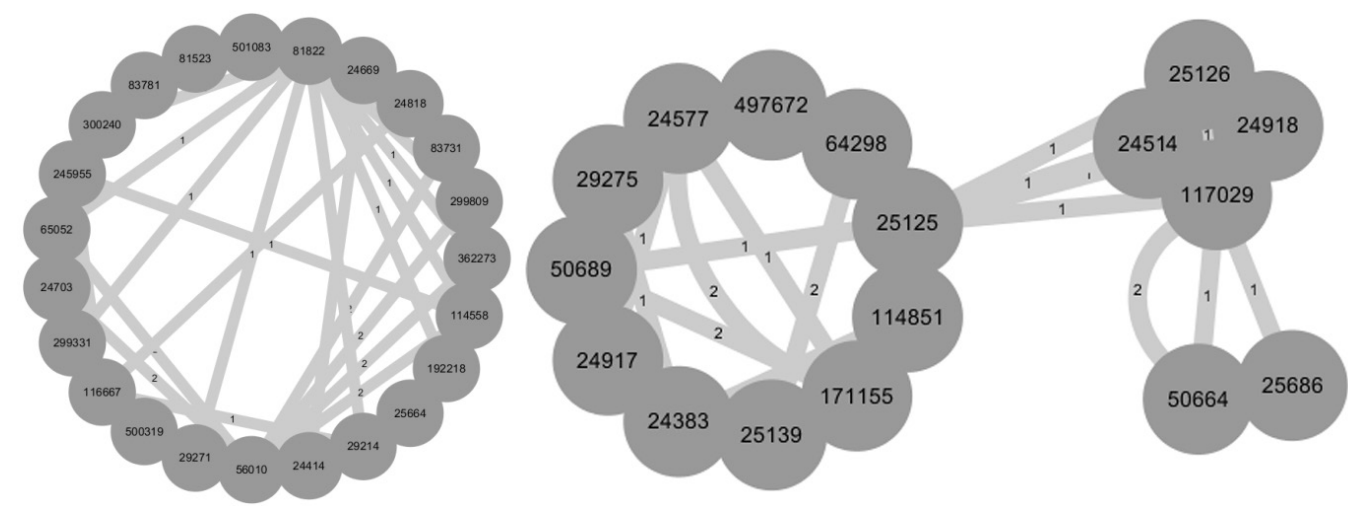

Figure 3. Modules mined from the interaction network at time point of $8 \mathrm{~h}$.

\section{Functional enrichment analysis results for modules}

The functions of modules were analyzed with NOA and P less than 0.05 was set as the cut-off. For module 1, cell surface receptor-linked signaling pathway (GO:0007166), response to wounding (GO:0009611) and signaling pathway (GO:0023060) were significantly overrepresented.

\section{DISCUSSION}

Changes in gene expression and subsequently in pathways occur during the progression of TBI. Systematic evaluation of the alterations is helpful in disclosing the mechanisms as well as identifying biomarkers. GSE2392 was first used to compare gene expression patterns 
of lateral fluid percussion-induced brain injury in rat and lateral controlled cortical impact injury in mice in 2003 (Natale et al., 2003), and the main results were the identification of candidate molecular functions and pathways induced by brain injury. In the present study, we combined differential analysis and module mining of the DEG interaction networks to analyze the microarray data of lateral fluid percussion-induced TBI in rats. Several modules were obtained at time points 8 and $72 \mathrm{~h}$. Functional enrichment analysis of the mined modules revealed that cell surface receptor-linked signaling pathway, response to wounding and signaling pathway were significantly changed. A range of interesting DEGs were also uncovered, which may be potential biomarkers.

Signal transducer and activator of transcription 3 (STAT3) is a member of the STAT protein family. It acts as a transcription activator in response to cell stimuli and thus plays a key role in many cellular processes such as cell growth and apoptosis (Grandis et al., 2000; Abell et al., 2005). Inhibition of STAT3 expression induces apoptosis in certain cancers, such as breast cancer (Kunigal et al., 2009) and prostate cancer (Mora et al., 2002). It's involvement in the protection and regeneration of neurons has been reported (Xi et al., 2003). Kretz et al. (2005) found that erythropoietin promotes regeneration of adult neurons via the activation of the Jak2/Stat 3 and PI3K/AKT pathways. It also regulates astrogliosis and scar formation after spinal cord injury (Herrmann et al., 2008). We found that it was upregulated at $8 \mathrm{~h}$, suggesting that apoptosis was repressed at that time. Maybe it can be used to monitor the renewal process of neurons.

Signal transducer and activator of transcription 5B (STAT5B) is another member of the STAT protein family and it was also upregulated at $8 \mathrm{~h}$ after TBI. It exhibits similar functions as STAT3 and is implicated in diverse biological processes, such as TCR signaling, apoptosis and adult mammary gland development. It is believed that constitutive activation of Stat5b contributes to carcinogenesis in vivo (Xi et al., 2003). Inhibition of STAT5b suppresses proliferation of human glioblastoma multiforme cells, and thus, it may serve as a therapeutic target (Liang et al., 2009). It can be activated by erythropoietin and thus mediates proliferation signaling (McPherson and Juul, 2007).

Chemokine (C-C motif) receptor 5 (CCR5) is a member of the beta chemokine receptor family. It is involved in inflammation (Qin et al., 1998; Bing et al., 2006) and thus plays a role in the pathogenesis of brain injury and neurodegenerative disorders (Whitney et al., 2009). It has been found to be upregulated in TBI (Schmidt et al., 2004) and this was confirmed in the present study. Galasso et al. (1998) reported that excitotoxic brain injury stimulates expression of the chemokine receptor CCR5 in neonatal rats. Its ligands include monocyte chemoattractant protein 2 (MCP-2) macrophage inflammatory protein 1 alpha (MIP-1 alpha), macrophage inflammatory protein 1 beta (MIP-1 beta), and so on. Besides, it can mediate the migration of mesenchymal stem cells to the impaired site in the brain (Ji et al., 2004a). It has been found that chemokine receptors are widely expressed in neural progenitor cells (Ji et al., 2004b). Carbonell et al. (2005) reported that pharmacological blockade of CCR5 reduces the migration velocity of perilesional microglia (Carbonell et al., 2005).

Janus kinase 2 (JAK2) is a protein tyrosine kinase. Its upregulation may be beneficial to the injured cortex; for example, it interacts with STAT3 and mediates the neuroprotection by erythropoietin (Kretz et al., 2005; Grasso et al., 2007).

Moreover, functional enrichment analysis was also conducted for DEGs at $4 \mathrm{~h}$. It showed that upregulated genes were enriched in terms of negative regulation of protein amino acid phosphorylation and phosphorus metabolic process, and downregulated genes were en- 
riched in the epoxygenase P450 pathway and several metabolic processes. Previous studies have indicated the close relationship between TBI and changes in amino acid neurotransmitters. Schumann et al. (2008) found that inhibition of NR2B phosphorylation restores alterations in N-methyl-D-aspartate (NMDA) receptor expression and improves functional recovery following TBI in mice. Spaethling et al. (2012) reported that NMDA receptor-mediated phosphorylation of GluR1 subunits contributes to the appearance of calcium-permeable AMPA receptors after mechanical stretch injury. Bhardwaj et al. (2000) concluded that P-450 epoxygenase and NO synthase pathways are involved in the local cerebral blood flow response to NMDA receptor activation. We found here that the epoxygenase P450 pathway was repressed, which is in accordance with previous findings.

\section{CONCLUSION}

Overall, differential analysis combined with module mining was adopted in the present study to identify TBI-related key modules. Functional enrichment analysis further described their biological functions in the progression of TBI. A number of novel and known significantly altered genes and pathways were identified. These findings provide directions for future research, and some of them can be developed into biomarkers for diagnosis or treatments.

\section{Conflicts of interest}

The authors declare no conflict of interest.

\section{ACKNOWLEDGMENTS} 81000859).

\section{REFERENCES}

Abell K, Bilancio A, Clarkson RW, Tiffen PG, et al. (2005). Stat3-induced apoptosis requires a molecular switch in PI(3) K subunit composition. Nat. Cell Biol. 7: 392-398.

Apweiler R, Bairoch A, Wu CH, Barker WC, et al. (2004). UniProt: the Universal Protein knowledgebase. Nucleic Acids Res. 32: D115-D119.

Bhardwaj A, Northington FJ, Carhuapoma JR, Falck JR, et al. (2000). P-450 epoxygenase and NO synthase inhibitors reduce cerebral blood flow response to N-methyl-D-aspartate. Am. J. Physiol. Heart Circ. Physiol. 279: H1616-H1624.

Bing M, Liu W, Homer RJ, Lee PJ, et al. (2006). Role of CCR5 in the pathogenesis of IL-13-induced inflammation and remodeling. J. Immunol. 176: 4968-4978.

Brown KR and Jurisica I (2005). Online predicted human interaction database. Bioinformatics 21: 2076-2082.

Brown KR and Jurisica I (2007). Unequal evolutionary conservation of human protein interactions in interologous networks. Genome Biol. 8: R95.

Carbonell WS, Murase S, Horwitz AF and Mandell JW (2005). Migration of perilesional microglia after focal brain injury and modulation by CC chemokine receptor 5: an in situ time-lapse confocal imaging study. J. Neurosci. 25: 70407047.

Costa IG, Schonhuth A and Schliep A (2005). The Graphical Query Language: a tool for analysis of gene expression timecourses. Bioinformatics 21: 2544-2545.

Di Giovanni S, Movsesyan V, Ahmed F, Cernak I, et al. (2005). Cell cycle inhibition provides neuroprotection and reduces glial proliferation and scar formation after traumatic brain injury. Proc. Natl. Acad. Sci. U. S. A. 102: 8333-8338.

Ernst J and Bar-Joseph Z (2006). STEM: a tool for the analysis of short time series gene expression data. BMC 
Bioinformatics 7: 191.

Ernst J, Nau GJ and Bar-Joseph Z (2005). Clustering short time series gene expression data. Bioinformatics 21 (Suppl 1): i159-i168.

Faden AI (2002). Neuroprotection and traumatic brain injury: theoretical option or realistic proposition. Curr. Opin. Neurol. 15: 707-712.

Galasso JM, Harrison JK and Silverstein FS (1998). Excitotoxic brain injury stimulates expression of the chemokine receptor CCR5 in neonatal rats. Am. J. Pathol. 153: 1631-1640.

Grandis JR, Drenning SD, Zeng Q, Watkins SC, et al. (2000). Constitutive activation of Stat3 signaling abrogates apoptosis in squamous cell carcinogenesis in vivo. Proc. Natl. Acad. Sci. U. S. A. 97: 4227-4232.

Grasso G, Sfacteria A, Meli F, Fodale V, et al. (2007). Neuroprotection by erythropoietin administration after experimental traumatic brain injury. Brain Res. 1182: 99-105.

Herrmann JE, Imura T, Song B, Qi J, et al. (2008). STAT3 is a critical regulator of astrogliosis and scar formation after spinal cord injury. J. Neurosci. 28: 7231-7243.

Ji JF, He BP, Dheen ST and Tay SS (2004a). Expression of chemokine receptors CXCR4, CCR2, CCR5 and CX3CR1 in neural progenitor cells isolated from the subventricular zone of the adult rat brain. Neurosci. Lett. 355: 236-240.

Ji JF, He BP, Dheen ST and Tay SS (2004b). Interactions of chemokines and chemokine receptors mediate the migration of mesenchymal stem cells to the impaired site in the brain after hypoglossal nerve injury. Stem Cells 22: 415-427.

Kretz A, Happold CJ, Marticke JK and Isenmann S (2005). Erythropoietin promotes regeneration of adult CNS neurons via Jak2/Stat3 and PI3K/AKT pathway activation. Mol. Cell Neurosci. 29: 569-579.

Kunigal S, Lakka SS, Sodadasu PK, Estes N, et al. (2009). Stat3-siRNA induces Fas-mediated apoptosis in vitro and in vivo in breast cancer. Int. J. Oncol. 34: 1209-1220.

Lei P, Li Y, Chen X, Yang S, et al. (2009). Microarray based analysis of microRNA expression in rat cerebral cortex after traumatic brain injury. Brain Res. 1284: 191-201.

Liang QC, Xiong H, Zhao ZW, Jia D, et al. (2009). Inhibition of transcription factor STAT5b suppresses proliferation, induces G1 cell cycle arrest and reduces tumor cell invasion in human glioblastoma multiforme cells. Cancer Lett. 273: 164-171.

Long Y, Zou L, Liu H, Lu H, et al. (2003). Altered expression of randomly selected genes in mouse hippocampus after traumatic brain injury. J. Neurosci. Res. 71: 710-720.

Maas AI, Stocchetti N and Bullock R (2008). Moderate and severe traumatic brain injury in adults. Lancet Neurol. 7 : 728-741.

McPherson RJ and Juul SE (2007). High-dose erythropoietin inhibits apoptosis and stimulates proliferation in neonatal rat intestine. Growth Horm. IGF Res. 17: 424-430.

Michael DB, Byers DM and Irwin LN (2005). Gene expression following traumatic brain injury in humans: analysis by microarray. J. Clin. Neurosci. 12: 284-290.

Mora LB, Buettner R, Seigne J, Diaz J, et al. (2002). Constitutive activation of Stat3 in human prostate tumors and cell lines: direct inhibition of Stat3 signaling induces apoptosis of prostate cancer cells. Cancer Res. 62: 6659-6666.

Natale JE, Ahmed F, Cernak I, Stoica B, et al. (2003). Gene expression profile changes are commonly modulated across models and species after traumatic brain injury. J. Neurotrauma 20: 907-927.

Opgen-Rhein R and Strimmer K (2007). From correlation to causation networks: a simple approximate learning algorithm and its application to high-dimensional plant gene expression data. BMC Syst. Biol. 1: 37.

Parikh S, Koch M and Narayan RK (2007). Traumatic brain injury. Int. Anesthesiol. Clin. 45: 119-135.

Qin S, Rottman JB, Myers P, Kassam N, et al. (1998). The chemokine receptors CXCR3 and CCR5 mark subsets of T cells associated with certain inflammatory reactions. J. Clin. Invest. 101: 746-754.

Rehman T, Ali R, Tawil I and Yonas H (2008). Rapid progression of traumatic bifrontal contusions to transtentorial herniation: A case report. Cases J. 1: 203.

Saeed AI, Sharov V, White J, Li J, et al. (2003). TM4: a free, open-source system for microarray data management and analysis. Biotechniques 34: 374-378.

Schafer J and Strimmer K (2005). An empirical Bayes approach to inferring large-scale gene association networks. Bioinformatics 21: 754-764.

Schmidt O, Infanger M, Heyde C, Ertel W, et al. (2004). The role of neuroinflammation in traumatic brain injury. Eur. J. Trauma 30: 135-149.

Schumann J, Alexandrovich GA, Biegon A and Yaka R (2008). Inhibition of NR2B phosphorylation restores alterations in NMDA receptor expression and improves functional recovery following traumatic brain injury in mice. $J$. Neurotrauma 25: 945-957.

Shannon P, Markiel A, Ozier O, Baliga NS, et al. (2003). Cytoscape: a software environment for integrated models of 
biomolecular interaction networks. Genome Res. 13: 2498-2504.

Smoot ME, Ono K, Ruscheinski J, Wang PL, et al. (2011). Cytoscape 2.8: new features for data integration and network visualization. Bioinformatics 27: 431-432.

Spaethling J, Le L and Meaney DF (2012). NMDA receptor mediated phosphorylation of GluR1 subunits contributes to the appearance of calcium-permeable AMPA receptors after mechanical stretch injury. Neurobiol. Dis. 46: 646-654.

Tusher VG, Tibshirani R and Chu G (2001). Significance analysis of microarrays applied to the ionizing radiation response. Proc. Natl. Acad. Sci. U. S. A. 98: 5116-5121.

Wang J, Huang Q, Liu ZP, Wang Y, et al. (2011). NOA: a novel Network Ontology Analysis method. Nucleic Acids Res. 39: e87.

Whitney NP, Eidem TM, Peng H, Huang Y, et al. (2009). Inflammation mediates varying effects in neurogenesis: relevance to the pathogenesis of brain injury and neurodegenerative disorders. J. Neurochem. 108: 1343-1359.

Xi S, Zhang Q, Gooding WE, Smithgall TE, et al. (2003). Constitutive activation of Stat5b contributes to carcinogenesis in vivo. Cancer Res. 63: 6763-6771.

Zhou B, Xu W, Herndon D, Tompkins R, et al. (2010). Analysis of factorial time-course microarrays with application to a clinical study of burn injury. Proc. Natl. Acad. Sci. U. S. A. 107: 9923-9928. 